\section{STATUS OF THE NEW ECRH SYSTEM FOR ASDEX UPGRADE}

D.Wagner ${ }^{1}$, G.Grünwald ${ }^{1}$, F.Leuterer ${ }^{1}$, F.Monaco ${ }^{1}, M$ Münich $^{1}, H$. Schütz ${ }^{1}$, F. Ryter ${ }^{1}$, R. Wilhelm ${ }^{1}$, H. Zohm ${ }^{1}$, T. Franke ${ }^{1}$, G. .Dammertz ${ }^{2}, H$. Heidinger $^{3}$, K.Koppenburg ${ }^{2}$, M.Thumm ${ }^{2}$, X. Yang ${ }^{2}$, W.Kasparek ${ }^{4}$, G.Gantenbein ${ }^{4}$, H.Hailer ${ }^{4}$, G.G. Denisov ${ }^{5}$, A.Litvak ${ }^{5}$, V. Zapevalov ${ }^{5}$

${ }^{1}$ Max-Planck-Institut für Plasmaphysik, EURATOM Ass., D-85748 Garching, Germany Forschungszentrum Karlsruhe, EURATOM Ass., D-76021 Karlsruhe, Germany ${ }^{2}$ Institut für Hochleistungsimpuls- und Mikrowellentechnik ${ }^{3}$ Institut für Materialforschung

${ }^{4}$ Institut für Plasmaforschung, Universität Stuttgart, D-70569 Stuttgart, Germany ${ }^{5}$ Institute of Applied Physics, RAS, 603600 Nizhny Novgorod, Russia

email: dietmar.wagner@ipp.mpg.de

The new ECRH system which is currently under construction at ASDEX Upgrade is aimed for a total power of $4 \mathrm{MW}$, generated by 4 gyrotrons, at a pulse length of $10 \mathrm{sec}$. A particular feature is the use of multifrequency gyrotrons, where the gyrotrons will work either at two frequencies $(105 / 140 \mathrm{GHz})$ or will be step-tunable in the range 105$140 \mathrm{GHz}$. The system should allow for central heating at different magnetic fields. It will also give more flexibility for the stabilization of neoclassical tearing modes (NTM) through the possibility of current drive on the high field side without changing the magnetic field and very localized feedback controlled power deposition with a narrow beamwidth by using a fast steerable launcher. The transmission line components are especially designed to cope for the large bandwidth of the system.

\section{Requirements}

The power deposition in the plasma is primarily determined by the magnetic field. For a single frequency ECRH system this has the consequence that for central heating the magnetic field is no longer a free parameter. However, for plasmas with different plasma currents or different equilibria, the magnetic field should be a free parameter in order to operate at a reasonable edge safety factor $\mathrm{q}(\mathrm{a})$. Furthermore, in a plasma with given parameters, some experimental program points, like suppression of neoclassical tearing modes, require to drive current on the high field side without changing the magnetic field. These requests can be satisfied if the gyrotron frequency is variable. In the experiments performed up to now in ASDEX Upgrade the available power was only $2 \mathrm{MW}$, of which 1.6 MW is injected into the plasma. This imposed a real limit for current drive, NTM stabilization or generation of internal transport barriers [1]. The requirement for the new system is therefore an installed power of $4 \mathrm{MW}$.
Since the current diffusion time in hot plasmas, like those with an internal transport barrier and $\mathrm{Te}>10 \mathrm{keV}$, is several seconds, we require a pulse duration of $10 \mathrm{sec}$ compatible with the limit of flat top discharges. A further requirement is the capability for very localized power deposition such that its center can be feedback controlled, for instance to keep it on a resonant q-surface. For this purpose we will install fast moveable mirrors.

\section{Gyrotrons}

The system will be constructed in several steps. The first gyrotron to be installed can work at $105 \mathrm{GHz}$ and at $140 \mathrm{GHz}$. The corresponding operating modes are $\mathrm{TE}_{17,6}$ and $\mathrm{TE}_{22,8}$ (two-frequency gyrotron). Here we make use of the $3 \lambda / 2$ and $4 \lambda / 2$ resonances ( $\lambda$ is the wavelength) of the single disk synthetic diamond vacuum window at these frequencies. A second gyrotron will be steptunable, i.e. it can work at several frequencies within the same frequency range. For each frequency the gyrotron oscillates in a different cavity mode. Fig. 1 shows the possible modes and frequencies. Short pulse test gyrotrons have demonstrated efficient generation of power in excess of $1 \mathrm{MW}$ at all these frequencies [2,3]. The frequency can be changed between two ASDEX Upgrade pulses and requires a resetting of the cryomagnetic field, the gun magnetic field and the collector magnetic field. There is one complicating feature with such a gyrotron: the output beam leaves the gyrotron window at slightly different angles and positions so that mirror adjustment in the matching optics unit is necessary. In order to limit the number of required phase correcting mirror sets we will choose 4 frequencies as our main operating modes.

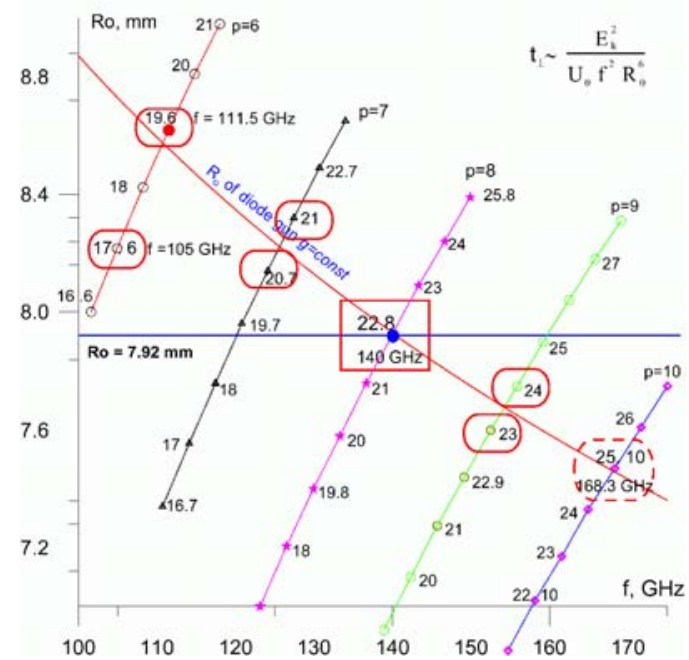

Figure 1. Electron beam radii and caustics for operating modes [3]. 


\section{Power Supplies}

All gyrotrons will have a single stage depressed collector. Therefore the beam voltage necessary for $1 \mathrm{MW}$ output power can be limited to a maximum value of $65 \mathrm{kV}$. The maximum beam current will be $40 \mathrm{~A}$. Since our power supplies can provide $80 \mathrm{~A}$ at $70 \mathrm{kV}$ we will run 2 gyrotrons from one power supply. There is a separate set of series tetrode and body modulators for each gyrotron (Fig.2), which will allow maximum flexibility for the experimental program.

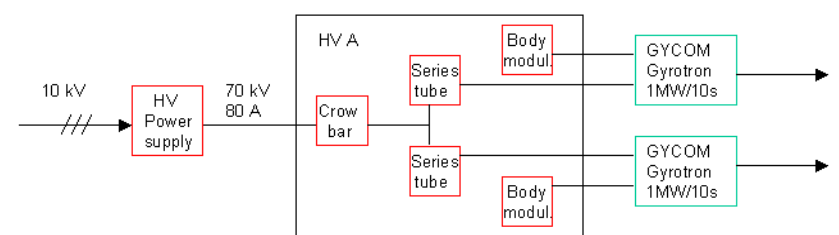

Figure 2. Schematic of power supply and modulator setup for 2 gyrotrons.

\section{Matching Optics Unit and Transmission Line}

The MOU (Fig.3) will contain different sets of phase correcting mirrors to match the gyrotron output beam at different frequencies to the transmission line input as well as broadband pulse monitoring and power measurement. One set of polarizers with groove depths of $\lambda / 4$ and $\lambda / 8$ at the center frequency of $122.5 \mathrm{GHz}$ proved to be sufficiently broadband to provide the required range of ellipticity for all necessary injection angles over the whole frequency band of the system and to avoid Wood's anomaly [5]. Figure 4 shows the calculated ellipticity of the output beam of the launcher in dependence of the polarizer angles for three different frequencies. The transmission will be in normal air, mainly through corrugated waveguides with I.D. $=87 \mathrm{~mm}$ over a total length of about $70 \mathrm{~m}$.

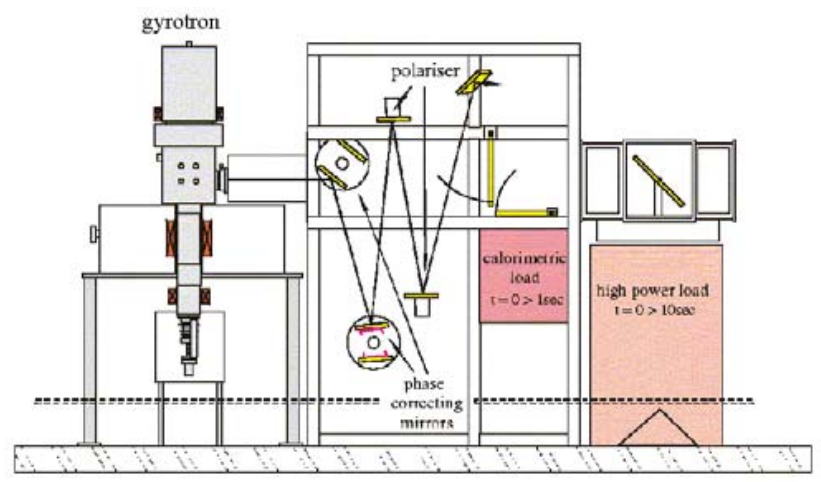

Figure 3. Gyrotron with matching optics unit (MOU) and calorimetric loads.
$105 \mathrm{GHz}$

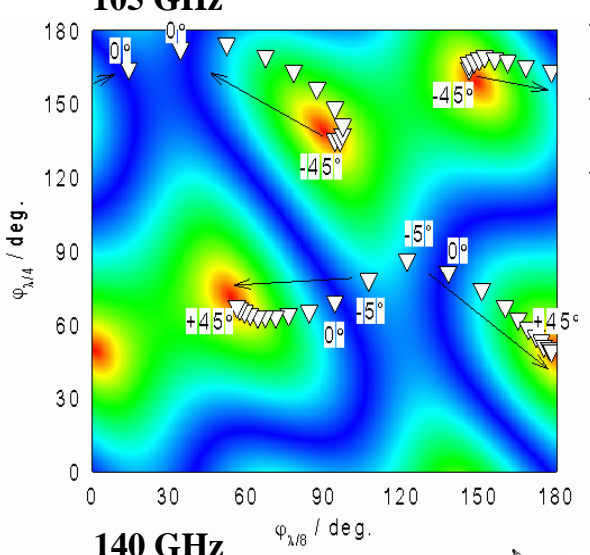

$140 \mathrm{GH}$ 90 deg.

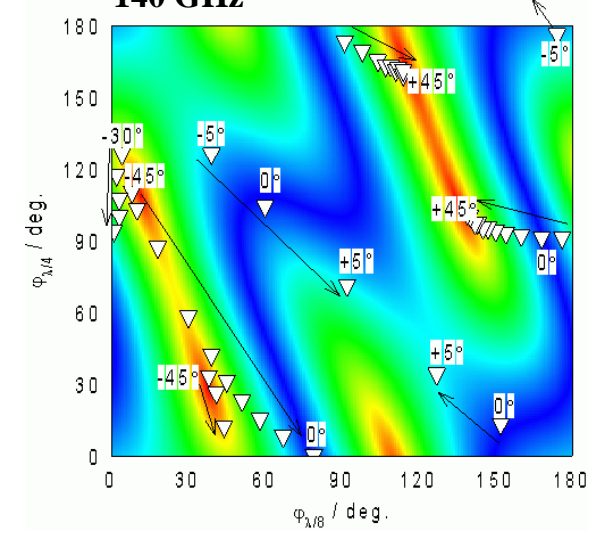

122.5 GHz

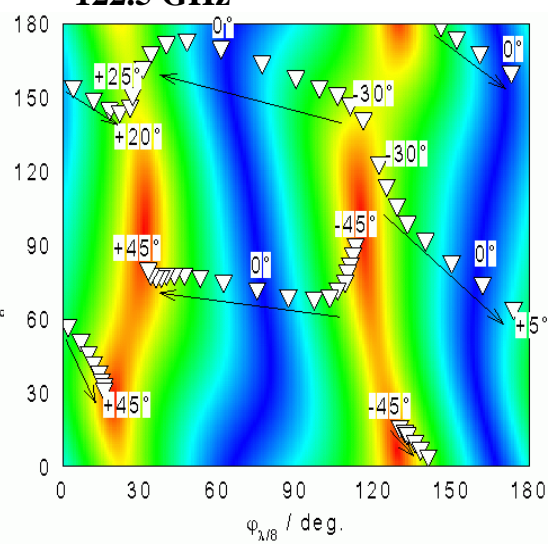

0.0

Figure 4. Calculated ellipticity of the broadband two-polarizer set. Indicated by the markers are the required ellipticities for a toroidal angular sweep at a constant poloidal angle of $10^{\circ}$ $\left(B_{t}=2.4 T, I_{p}=800 \mathrm{kA}\right)$.

\section{Broadband Window}

The torus window required for the step-tunable gyrotron should be in principle broadband, e.g. a Brewster window. To avoid constraints with respect to polarisation, a tunable double disk window with a remote controlled adjustment of the distance between the disks will be used (Fig.5). Two diamond disks with a thickness of $1.8 \mathrm{~mm}$ will be used for this window, where the disks

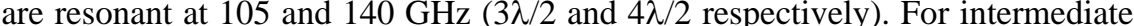
frequencies the double disk window can be tuned to a reflection minimum by changing the distance between the two disks. Figure 6 shows the calculated reflection for different distances between the disks. A critical value is the width of the Fabry-Perot resonances at intermediate frequencies between the single disk resonances. Only a maximum distance of $10 \mathrm{~mm}$ between the disks can be 
allowed for a possible frequency drift of $100 \mathrm{MHz}$ during the gyrotron pulse. For the two-frequency gyrotrons a single disk window is sufficient.

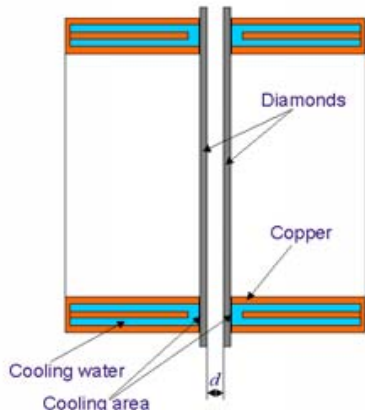

Figure 5. Tunable double disk window.

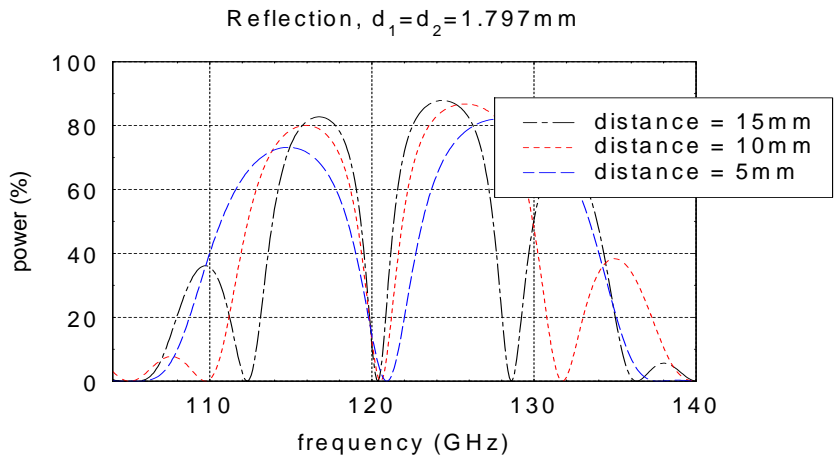

Figure 6. Calculated reflection of the double disk window for different distances between the disks.

\section{Fast Steerable Launcher}

A steerable launcher will enable to steer the beam over the whole plasma cross-section. In order to cope with the thermal load, disruption forces and the mechanical dynamics of the fast poloidal steering, the mirror will be made out of copper plated graphite. Two different types of drives will be used for the launcher. A slow drive to rotate the launcher around its axis on a shot to shot basis, mainly to set the toroidal launching angle and a fast drive to control the poloidal launching angle. Figure 7 shows the principle of the launcher drive. First test results are plotted in Fig.8. The design value of $10^{\circ} / 100 \mathrm{msec}$ was achieved.

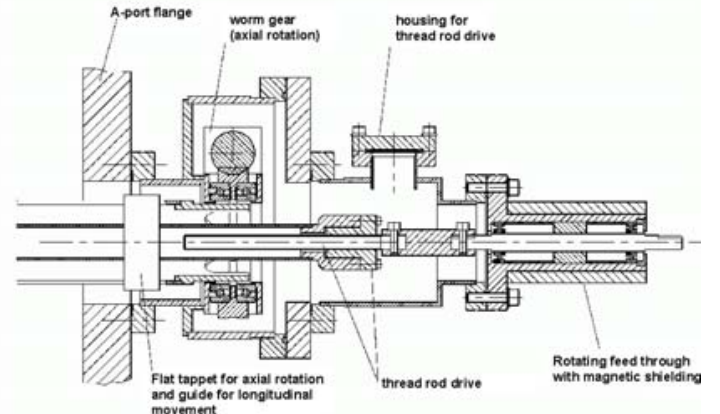

Figure 7. Fast steerable launcher drive.

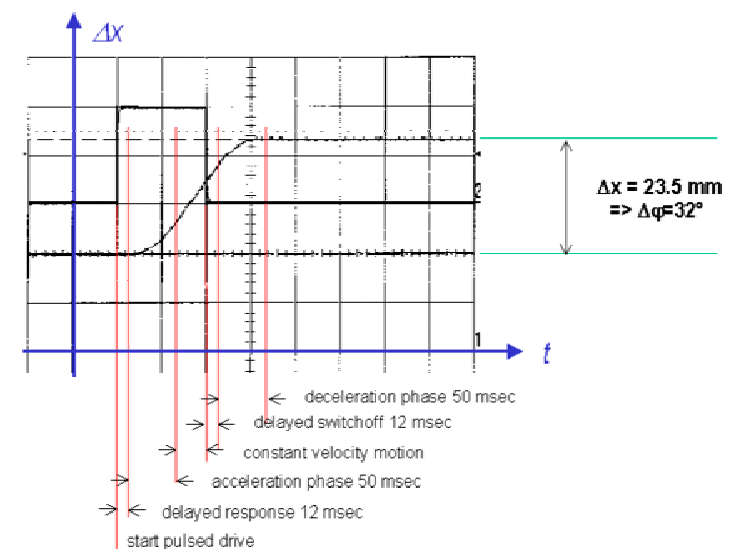

Figure 8. Measured poloidal angular variation of the fast steerable launcher.

\section{Summary}

The construction of a new step-tunable ECRH system is in progress at ASDEX Upgrade. The installation of two transmission lines with matching optics units (MOU) for the first two gyrotrons is almost finished. The power supplies for these gyrotrons have been successfully installed and tested.

\section{References}

[1] Leuterer F., et al., Fus. Eng. Des. 53 (2001) 277

[2] Thumm M., et al., Fus. Eng. Des. 53 (2001) 407

[3] Zapevalov V., $12^{\text {th }}$ Worksh. ECE and ECRH, Aix en Provence, France, 2002

[4] Ellis R., et al., AIP Conf. Proc. 595 (2001) 318

[5] Holzhauer E., 27 ${ }^{\text {th }}$ Int. Conf. IR and mm Waves, San Diego (USA), 2002

[6] Yang X., et al., Int. J. on Infrared and Millimeter Waves, 24 (2003) 1805 